\title{
Measurement and Control of Ultrashort Optical Pulse Propagation in Metal Nanoparticle-Covered Dielectric Surfaces
}

\author{
Jess M. Gunn, Scott H. High, Vadim V. Lozovoy, and Marcos Dantus* \\ Department of Chemistry, Michigan State University, East Lansing, Michigan 48824
}

Received: June 5, 2009; Revised Manuscript Received: June 1, 2010

\begin{abstract}
In this paper, we present a detailed study of localized two-photon photoluminescence from metal nanoparticles on a dielectric surface. An ultrafast ( $\sim 13 \mathrm{fs}$ ) near-infrared laser focused on a micrometer sized region is used to excite a silver nanoparticle covered quartz or mica substrate, and a pulse shaper is used to control and change the spectral phase of the excitation pulse, as well as allow for autocorrelation-type measurements. Spectra of the observed photoluminescence from individual localized regions remote from the focal spot are obtained. The spatial distribution of nonlinear photoluminescence is found to have characteristics very distinct from that of normal scatter. It is found that the excitation pulse propagates to the different remote regions by means of surface plasmon polariton (SPP) propagation. As the SPP propagates it accumulates different amounts of quadratic and cubic dispersion depending on the path taken. The quadratic and cubic dispersion for a number of such pathways is measured, and evidence of negative dispersion is observed. Photoluminescence is observed at locations where constructive interference and a localized resonance occurs, tens of micrometers from the incident laser pulse.
\end{abstract}

\section{Introduction}

Gold and silver nanoparticles have been the subject of numerous studies, given their plasmon resonance photoluminescent properties..$^{-5}$ In particular, the nonlinear optical properties of these nanoparticles have been the subject of great interest; we highlight studies of the two-photon-absorption-induced photoluminescence of metal nanoparticles,${ }^{6-8}$ and note that the efficiency of multiphoton excitation is so high for silver and gold nanoparticles that it has been detected for continuous wave laser excitation. ${ }^{9}$ Noble metal nanoparticles and rough surfaces have also been of interest due to their ability to enhance local electromagnetic fields, providing an enhancement in the twophoton excitation or hyper-Raman excitation of molecules. ${ }^{10-19}$

Also of much interest has been the ability to control the propagation of optical pulses as surface plasmon polaritons (SPP), with applications in the fields of plasmonics and nanophotonics, where specially constructed waveguides and optical devices bridge the realms of electronics and photonics. ${ }^{20-37}$ It was first proposed in 1981 that thin layers of silver could support surface plasmon propagation over a distance of $300 \mu \mathrm{m}$, an order of magnitude greater than thicker films. ${ }^{38}$ Numerous experiments have confirmed this work, indirectly and directly determining the propagation distance of an SPP over tens of micrometers, after which it localizes. ${ }^{39-46}$ A transition from localized surface plasmon resonance, the phenomenon by which individual metal particles enhance electromagnetic fields, to extended SPP has been found to occur in films of silver nanoparticles when their density approaches the percolation limit. ${ }^{47,48}$ This suggests that silver nanoparticle films near and above this limit are capable of sustaining long-range SPP transport. Of particular interest is the finding that near the percolation limit, sputtered Ag films (composed of Ag nanoparticles) exhibit the largest nonlinear absorption coefficient. ${ }^{49}$

* To whom correspondence should be addressed. E-mail: dantus@msu.edu. Phone: 517-355-9715 × 314. Fax: 517-353-1793.

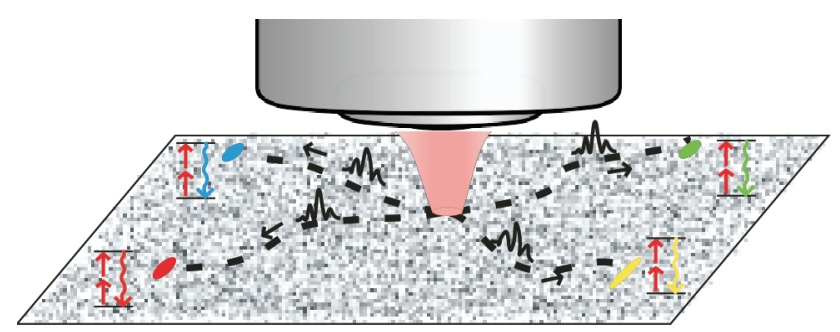

Figure 1. Schematic of energy transport. A high numerical-aperture objective excites an SPP on the surface of a highly interconnected silver nanoparticle film. Energy transport occurs over tens of micrometers before localizing and resulting in two-photon-induced photoluminescence.

In 2006, Gunn et al. reported the unprecedented detection of two-photon photoluminescence at distances greater than 100 times the focal spot diameter of the femtosecond laser used to excite a metal nanoparticle covered dielectric surface.$^{50}$ That work, which combined long-range SPP transport in silver films with the nonlinear optical properties of nanoparticles and rough surfaces discussed previously, showed that when an ultrashort pulse centered at $800 \mathrm{~nm}$ is focused to a $1 \mu \mathrm{m}$ diameter at normal incidence on a film of polydisperse silver nanoparticles, discrete localized two-photon-induced visible photoluminescence is observed up to $99 \mu \mathrm{m}$ from the focal spot (see schematic in Figure 1). The nonlinear photoluminescence was shown to be both polarization-dependent and polarized, although the polarization of the incident beam is not necessarily conserved. Furthermore, the intensity of photoluminescence in each region was found to depend on the spectral phase of the laser pulse used for excitation; this observation motivated the present, more rigorous, study using shaped femtosecond laser pulses.

This paper reports on further detailed exploration of the twophoton-induced photoluminescence observed at locations that are far from the region of excitation in films of $\mathrm{Ag}$ nanoparticles. In particular, spectra of the photoluminescence from the individual regions, which are in agreement with the two-photon 

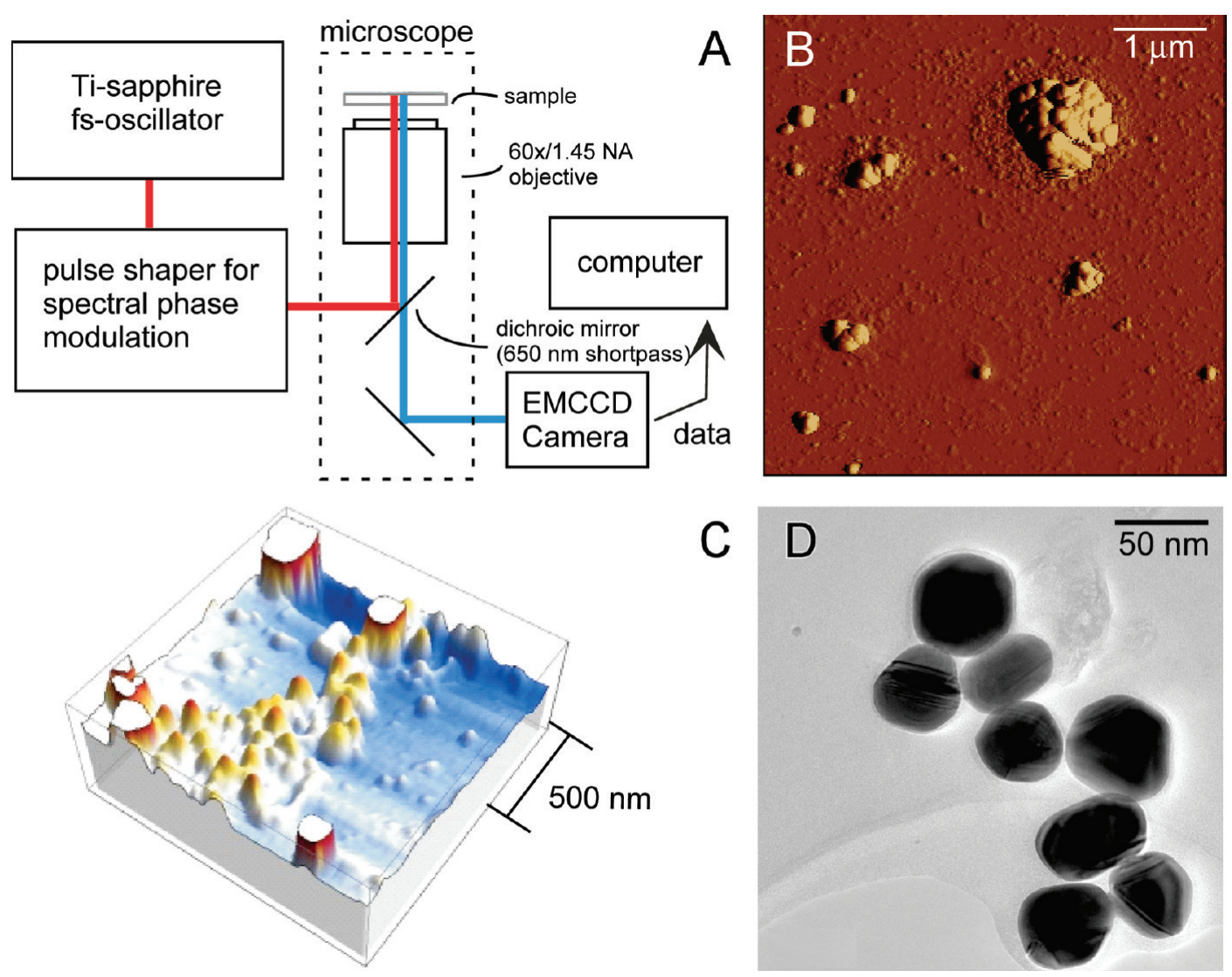

Figure 2. Panel A: Schematic of experimental setup. Panel B: AFM image of a typical sample, illustrating the larger features of the sample. Panel C: AFM image of a typical sample, with an emphasis on the smaller, more connected features that lie in between the larger features. Panel D: TEM image of silver nanoparticles from a typical sample.

photoluminescence from silver nanoparticles in $\mathrm{TiO}_{2},{ }^{51}$ and experiments to demonstrate that the reported emission has characteristics very distinct from laser-scattered light, are shown. Additionally, experiments using shaped femtosecond pulses to elucidate why certain phases cause strong photoluminescence from some regions and not others are reported. Finally, we perform experiments inspired by pump-probe measurements on photoelectron emission from silver nanoparticles, ${ }^{52-56}$ in which we report two-photon interferometric autocorrelation measurements made on individual photoluminescent regions.

\section{Experimental Section}

The experimental setup is shown in Figure 2A. Experiments are carried out with a Ti:sapphire femtosecond laser (13 fs, $\lambda_{0}$ $=805 \mathrm{~nm}, 85 \mathrm{~nm}$ fwhm) coupled to an inverted microscope with a $60 \times, 1.45$ NA objective to focus the beam onto the sample at normal incidence. The use of a high numerical aperture objective, in conjunction with the roughness of the sample, which is described in detail below, allows for momentum matching between light and the launched SPPs and makes propagation possible. ${ }^{57}$ The pulse energy is attenuated to $\sim 11$ $\mathrm{pJ} /$ pulse, resulting in an estimated energy density of $1.5 \mathrm{~mJ} /$ $\mathrm{cm}^{2}$ and a peak power density of $1.5 \times 10^{11} \mathrm{~W} / \mathrm{cm}^{2}$. At this high peak intensity, we find that unless energy transport is efficient, damage, likely melting, occurs near the focal spot, indicated by very unstable photoluminescence. In contrast, efficient energy transport, which is accompanied by low light emission at and near the laser focus, results in signals well outside the laser focus that can be stable for tens of minutes. Laser-scattered light $(700-900 \mathrm{~nm})$ is blocked by the use of a short-pass $(650 \mathrm{~nm})$ dichroic mirror in the setup, and wide- field images of emitted light are collected by an electron multiplier CCD camera. A $4 f$ pulse shaper incorporating a liquidcrystal spatial light modulator is used to control the spectral phase of the laser pulses, and compensation for pre-existing spectral phase distortions at the focal plane of the objective lens is accomplished using multiphoton intrapulse interference phase scan (MIIPS) (see the Supporting Information for more detail). ${ }^{58}$ All measurements are made at room temperature under ambient conditions.

The samples studied are a thin layer of polydisperse silver nanoparticles allowed to aggregate and deposit onto a quartz or mica substrate, prepared as described by Perry. ${ }^{59}$ AFM and TEM images of a typical sample are shown in Figure 2. Previously obtained SEM and TEM images of the particles show variation in their aspect ratio; the short dimension is typically $30-60 \mathrm{~nm}$, and the long dimension $30-200 \mathrm{~nm} .{ }^{50}$ As shown in panels B and C of Figure 2, the dielectric surface is only partially covered. There are a few large (micrometer) agglomerations of nanoparticles (seen prominently in Figure 2B), as well as interconnections among islands, observed in the form of regions with nanoparticles at distances shorter than the incident laser wavelength, shown in Figure 2C.

As discussed in more detail by Gunn et al., ${ }^{50}$ when the nearIR ultrashort pulses are incident on a solution of silver nanoparticle clusters similar to those deposited on the surface, broadband photoluminescence in the visible spectral region is observed, and demonstrates a quadratic dependence on the incident laser power. This photoluminescence is consistent with multiphoton-absorption mediated photoluminescence. ${ }^{6,7,60-62}$ 


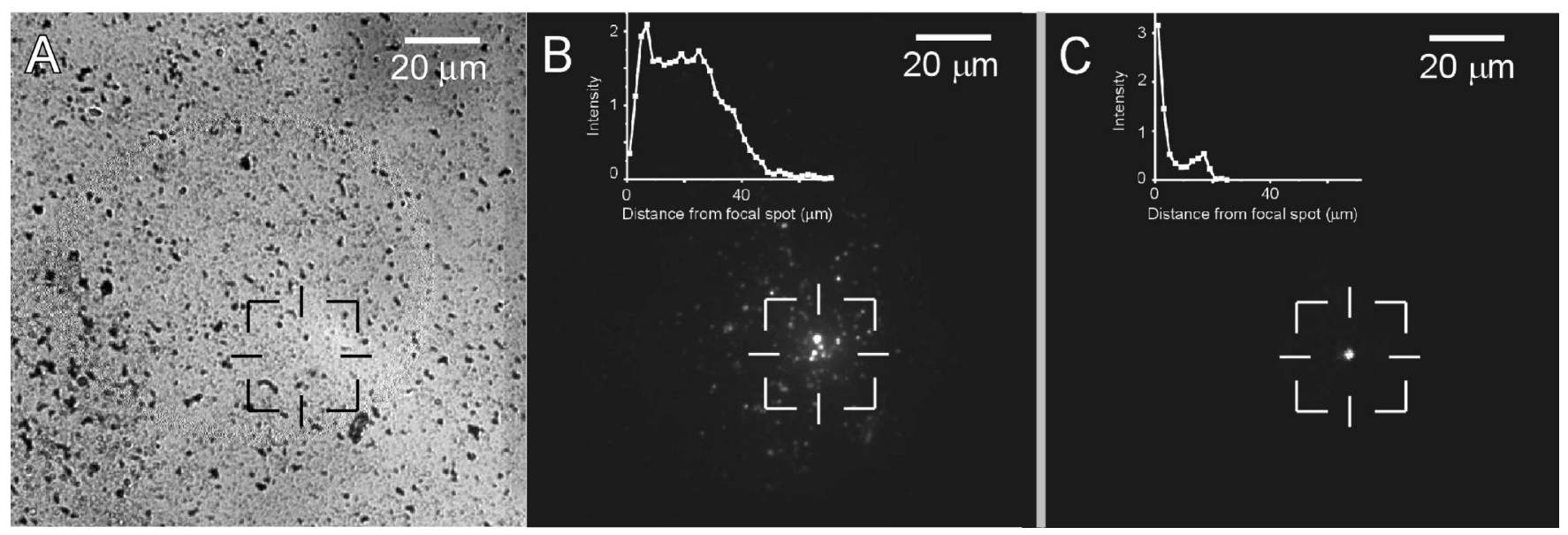

Figure 3. Images of a single sample under different types of illumination. Panel A shows the sample under white-light illumination. Panel B shows the sample excited at the crosshairs by a 13-fs Ti:sapphire laser. Panel C shows the sample excited at the crosshairs by a HeNe laser. The insets in panels B and C show the total intensity of all pixels with signal above the noise level as a function of distance from the focal spot. It is clear that the behavior observed upon excitation with a fs-laser is not consistent with the behavior of scatter observed upon excitation with a HeNe laser.

\section{Results/Discussion}

An image collected under white light illumination is shown in Figure 3A. The image shown in Figure 3B corresponds to the same sample shown in Figure $3 \mathrm{~A}$ when it is excited by ultrashort, near-IR, laser pulses focused down to a $1 \mu \mathrm{m}$ spot size located at the cross-hairs. This image was collected by the EMCCD camera under wide field conditions without scanning either the sample or the laser, and is representative of hundreds of images of dozens of samples that have been prepared with both quartz and mica as substrates. Discrete regions of photoluminescence up to tens of micrometers ( $>10$ focal diameters) from the excitation point are observed, and a power study of the dependence of photoluminescence intensity from these regions upon excitation power confirms earlier results indicating the process depends quadratically on the incident laser intensity. The inset in Figure 3B shows the total photoluminescence from the sample as a function of distance from the excitation point, in $2 \mu \mathrm{m}$ increments. As a comparison, the same sample was illuminated by a HeNe laser at $632 \mathrm{~nm}$, which was configured to propagate collinearly through the same optics, and thus focus at the same point. This wavelength is short enough $(<650 \mathrm{~nm})$ that it is not blocked by the dichroic mirrors and filters that prevent the observation of scattered light from the fs-laser, and allows characterization of the behavior of scattered light in this sample. As in Figure 3B, the inset in Figure 3C shows the total photoluminescence from the sample as a function of distance from the excitation point in $2 \mu \mathrm{m}$ increments. It is clear that the remote regions of photoluminescence observed upon excitation by the fs-laser (Figure 3B) are not consistent with the scattering behavior observed upon excitation with the $\mathrm{HeNe}$ laser (Figure 3C).

To further characterize the behavior of the emitted light, and compare what is observed upon excitation with a fs-laser to the scatter observed upon excitation with a HeNe laser, the intensity of $2-\mu \mathrm{m}$-wide cross sections running horizontally and vertically through the focal spot is plotted in Figure 4. From this figure, it is strikingly clear that the behavior observed upon excitation with the fs-laser is not scatter. The overall profile of the spatial distribution of the photoluminescence is much broader, with clearly discrete regions of emission (indicated by sharp spikes). When scatter is detected, no such discrete regions are observed. Another significant observation is that the maximum intensity for the fs-laser excited sample is not found
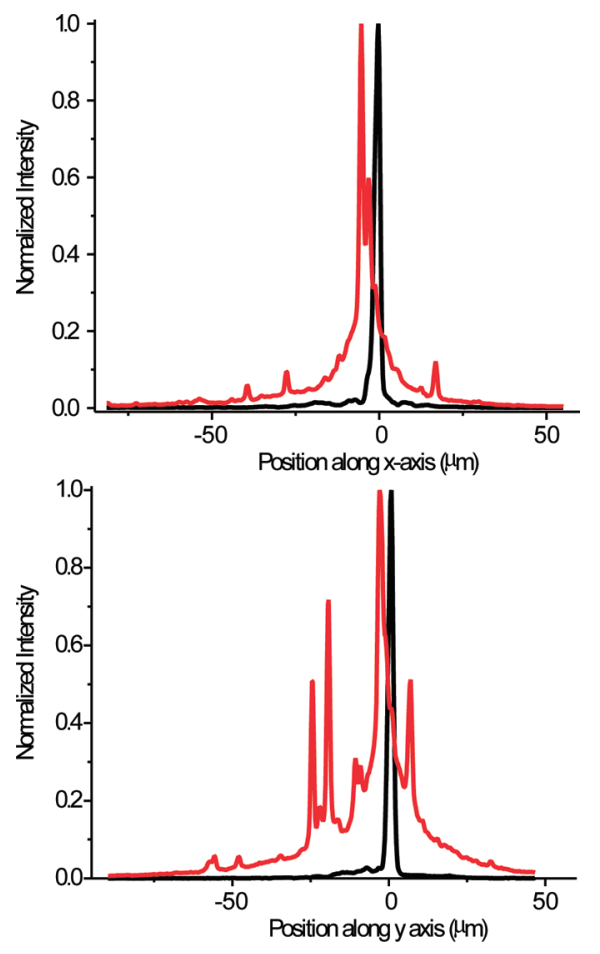

Figure 4. Intensity profiles for a $2-\mu \mathrm{m}$-wide section of data running horizontally (top) and vertically (bottom) through the focal spot for a sample excited by a HeNe laser (black) and a fs-laser (red).

immediately at the focal spot. This is consistent with numerous observations in our lab, in which very strong emission at the focal spot correlated with a lack of discrete regions of remote photoluminescence, i.e., strong emission at the focal spot, is indicative of poor energy transport.

Note that under fs-excitation, two-photon emission is seen over $40 \mu \mathrm{m}$ from the focal spot, consistent with the reports of tens of micrometers range of propagation of surface plasmons in metals on dielectric surfaces. ${ }^{39,43}$ This suggests that a surface covered by silver nanoparticles can behave as a continuous thin metallic surface under certain conditions. The dynamics of SPP transport on a dielectric surface partially covered by a very thin layer of nanoparticles ${ }^{2,47,63}$ and "islandized" metals ${ }^{42,64-67}$ are extensively discussed in the literature, and there is also evidence that the presence of relatively large, irregular clusters of 

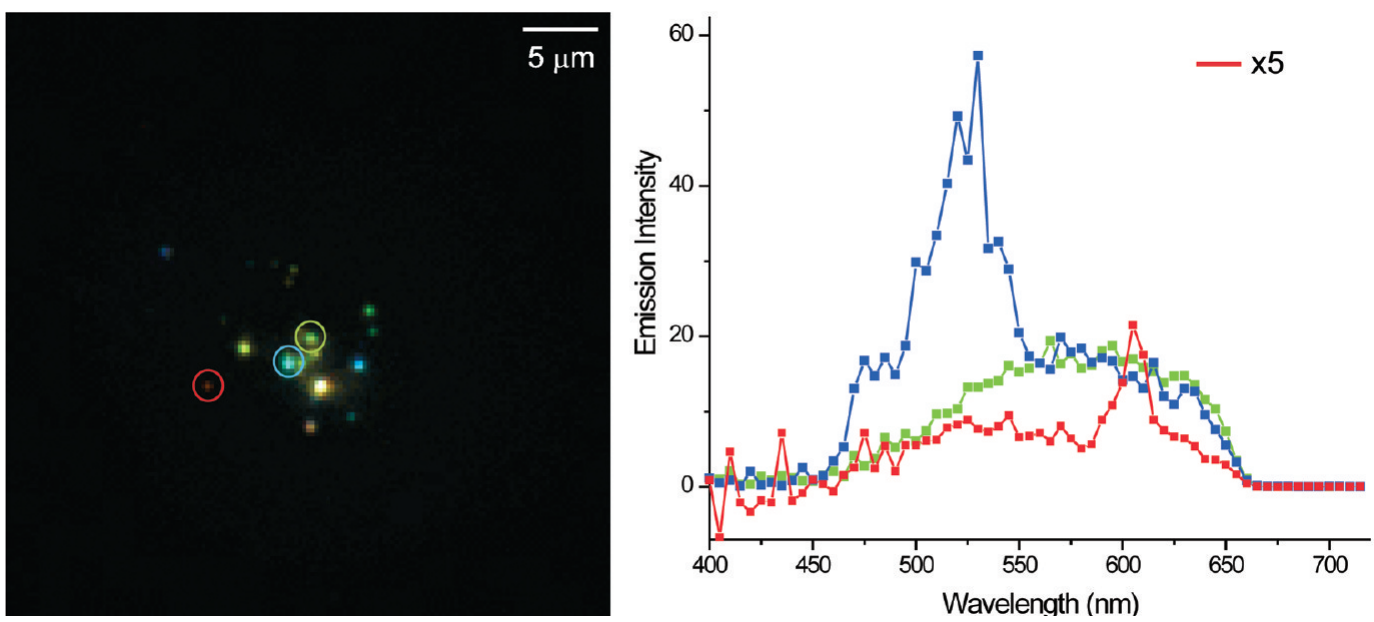

Figure 5. Spectral information for photoluminescence from individual regions of emission. The image on the left is a false color composite created by coloring photoluminescence between 450 and $525 \mathrm{~nm}$ blue, 525 and $600 \mathrm{~nm}$ green, and 600 and $675 \mathrm{~nm}$ red. The right panel shows the spectrum of the photoluminescence from the three circled regions. The photoluminescence from the red region is multiplied by five to be clearly visible on the same intensity axis.

nanoparticles is favorable for transition from a delocalized SPP mode to a localized surface plasmon. ${ }^{2,68,69}$ While the energy can be transported as either a near-IR wave $(\sim 800 \mathrm{~nm})$ or as a second harmonic $(\mathrm{SH})$ wave $(\sim 400 \mathrm{~nm})$, the IR SPP wave would be expected to have less attenuation than its corresponding $\mathrm{SH}$ wave. ${ }^{19,43,46,70,71}$

Further insight into the optical properties of the sample is gained by placing an electronically controlled variable color filter (VariSpec Filter 51010, CRi) immediately before the camera. This device allows only a narrow band of wavelengths $(10 \mathrm{~nm}$ fwhm) to pass. By scanning the transmission wavelength, a spectrum of the photoluminescence can be acquired. A false color composite image is shown in Figure 5, left. Photoluminescence from $450-525 \mathrm{~nm}$ is blue, $525-600 \mathrm{~nm}$ green, and 600-675 $\mathrm{nm}$ red. It is clear that different regions of emission have distinct wavelengths of photoluminescence. Figure 5, right, shows the spectra of the three circled regions. From these spectra, several properties of the photoluminescence can be determined. First, there is no photoluminescence below $450 \mathrm{~nm}$, so the observed photoluminescence cannot be $\mathrm{SH}$ generation of the fundamental excitation beam. Second, there is no photoluminescence above $660 \mathrm{~nm}$, showing that the dichroic mirror used to separate the excitation from the photoluminescence is effective, and that the observed photoluminescence cannot be attributed to direct scatter of the fundamental of the excitation laser beam. Third, the spectra illustrate that the regions have photoluminescences with different line shapes and line widths. The region of red photoluminescence has a narrow emission around $610 \mathrm{~nm}$, while the green photoluminescence is very broad. Composite images from additional samples are included in the Supporting Information. These spectra are in good agreement with previously reported two-photon photoluminescence spectra of thin films of Ag nanoparticles in dielectric media. ${ }^{51,72,73}$

To investigate the nature of energy transport in these samples, the pulse shaper was programmed to produce a pair of pulses and to scan the delay between them. ${ }^{74,75}$ Although similar types of experiments have been reported in the literature, ${ }^{52-55,76}$ our experiments are different from those because in our case the excitation is in the near-IR, which then propagates, and the measured signal corresponds to localized two-photon-induced photoluminescence and not to photoelectrons, as, for example in ref 77 .
Figure 6 shows the nonlinear photoluminescence from individual regions of the thin film as a function of time delay between the two pulses. Because the two pulses are collinear, they interfere with each other. The top panel shows the signal obtained from a frequency doubling crystal for reference. The 2.67 fs oscillations correspond to the optical period of the fundamental laser wavelength. A representative image is shown at left, and the intensities of the three circled points, as a function of the delay between pulses, are shown to the right.

Over the course of the autocorrelation, it has been observed that some regions will spontaneously stop emitting. Of these, some will later be observed to emit with the same properties observed before, in a blinking phenomenon. Others will remain "off". The behavior of those regions, and others that exhibit an irreversible change in behavior, such as region a, is attributed to structural changes to the sample upon irradiation, with melting of the sample a likely cause. It is significant to note that adjacent regions, such as a and $b$, which are separated by approximately $1 \mu \mathrm{m}$, can exhibit very different behaviors, as shown in Figure 6. In some cases, such as region a, the photoluminescence exhibits an abrupt change in intensity. These changes can be seen as an increase or decrease in intensity. Other regions, such as $b$, behave symmetrically, and remarkably similar to the second-harmonic signal provided for comparison. This indicates that the regions respond independently to the laser used for illumination, and suggests that no sequential scattering is taking place. Additionally, the correspondence of the period and overall shape of photoluminescence from this region with secondharmonic signal is further evidence that the behavior observed is two-photon in nature.

Other behaviors are also observed in the optical response, including broadening (region c) of the pulse, which indicates that quadratic dispersion has been acquired as the SPP propagates. Side peaks, visible in the signal from region $b$ and more pronounced in other regions, suggest that cubic dispersion is also acquired by the excitation pulse as it propagates to the regions where localized two-photon photoemission occurs.

Clearly, there are significant differences in the luminescent properties of different regions of the sample while an autocorrelation is performed at the focus. While the implications of some of these observations are not immediately clear, the observed broadening and presence of side peaks in the traces are consistent with the behavior expected if the pulse experi- 

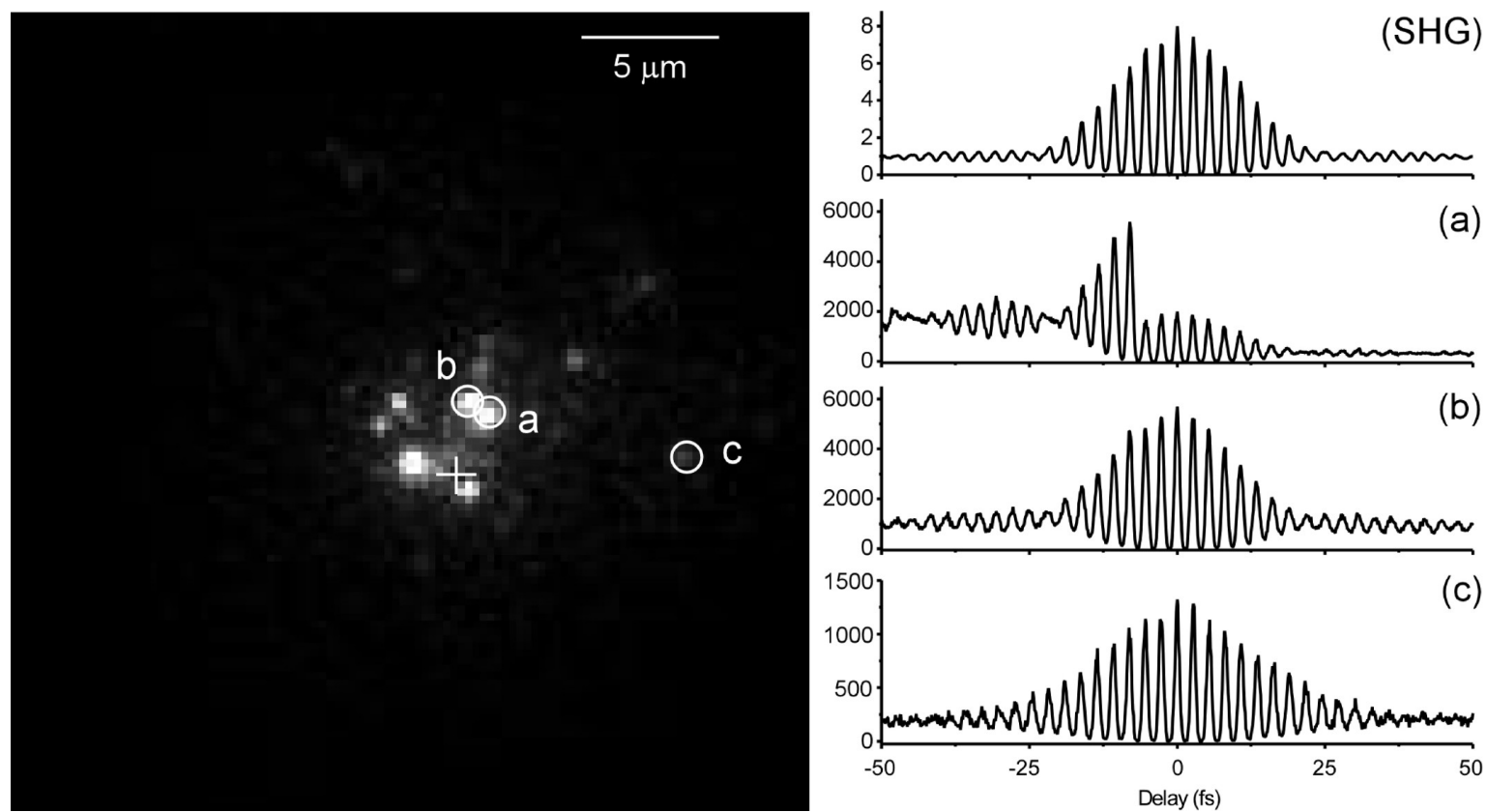

Figure 6. Photoluminescence from several discrete regions is shown as a pulse shaper is used to produce a pair of pulses and scan the delay between them. The top panel shows the optical response of a frequency doubling crystal as a function of delay between pulses. Panels a, $b$, and $c$ show the optical response from different regions. Some regions show broadening, as shown in region c. Others show wings (visible in panel b), or erratic behavior, likely due to melting or other irreversible change to the sample, as shown in panel a. The behaviors are independent of distance from the excitation point, as well as the behavior of neighboring regions (compare regions a and $\mathrm{b}$, which are only separated by approximately 1 $\mu \mathrm{m})$.

enced dispersion as it propagated through the sample. As each region of photoluminescence results from energy traveling along a different path, it would be expected to find evidence for pulse broadening due to dispersion (chirp). We note that this observation has fundamentally different physics than the observation of electronic dephasing on single nanoparticles. ${ }^{52-56}$ Given that the group velocity of an SPP is slower than the speed of light, ${ }^{78-81}$ the time required for the energy to propagate between the excitation point and the bright point of photoluminescence must be many times longer than the pulse duration $(\sim 13 \mathrm{fs})$; we conclude that the SPP propagates long $(\sim 100 \mathrm{fs})$ after the laser pulse excitation. Pulse broadening in our measurements indicates that the propagating SPP experiences different amounts of dispersion as it travels to the region where it induces twophoton photoluminescence, a concept we explore further below.

Theory has suggested that pulse shaping could be used as a mechanism to control plasmon propagation, ${ }^{82-86}$ and preliminary experiments have indicated that localization of plasmon emission on a nanometer scale is possible..$^{55,76,87,88}$ The previous work of Gunn et al. ${ }^{50}$ showed that the discrete regions of emission are sensitive to the spectral phase of the laser. In that work it was shown that pulse shaping controlled localized photoluminescence over distances of tens of micrometers.

As suggested by the autocorrelation data in Figure 6, the region-dependent sensitivity to spectral phase may be due to the acquisition of dispersion by the pulse as it propagates through the sample. To directly quantify the amount of quadratic and cubic dispersion acquired by the pulse as it propagates to each region, we applied a grid of phase functions, based on the Taylor expansion of the spectral phase, of the form $\varphi\left(\phi^{\prime \prime}, \phi^{\prime \prime \prime}\right)$ $={ }^{1} / 2\left[\phi^{\prime \prime}\left(\omega-\omega_{0}\right)^{2}\right]+{ }^{1} / 6\left[\phi^{\prime \prime \prime}\left(\omega-\omega_{0}\right)^{3}\right]$ to the excitation pulse, where $\phi^{\prime \prime}$ and $\phi^{\prime \prime \prime}$ correspond to the quadratic and cubic dispersion, and monitored the intensity of two-photon-induced photoluminescence from discrete regions on the sample. Assuming that maximum photoluminescence from a particular region occurs when the pulse is transform-limited $\left(\phi^{\prime \prime}=\phi^{\prime \prime \prime}=\right.$

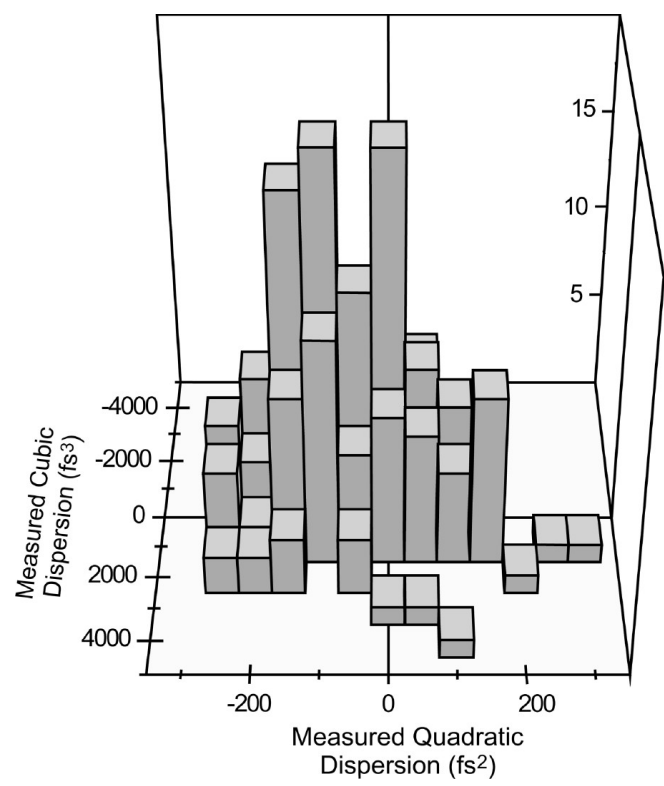

Figure 7. A 3-dimensional histogram of measured values for $\phi^{\prime \prime}{ }_{\mathrm{w}}$ and $\phi^{\prime \prime}{ }_{\mathrm{w}}$ for over 200 regions of photoluminescence on a single sample. The bins correspond to the step size used for $\phi^{\prime \prime}{ }_{\mathrm{w}}$ and ${\phi^{\prime \prime \prime}}_{\mathrm{w}}$, which in turn were chosen based on the experimental error in a given measurement.

0 ), we can directly determine the chirp introduced: $\phi^{\prime \prime}{ }_{\mathrm{w}}=$ $-\phi^{\prime \prime}{ }_{\max }$ and $\phi^{\prime \prime \prime}{ }_{\mathrm{w}}=-\phi^{\prime \prime \prime}{ }_{\max }$. Validation for this assumption is presented in the Supporting Information.

For each experiment, a collection of 441 images were acquired, each one with values for $\phi^{\prime \prime}$ and $\phi^{\prime \prime \prime}$ defined by the grid discussed above. Figure 7 summarizes the results for these measurements after tracking the phase dependence of $\sim 200$ individual regions of localized photoluminescence. The 3dimensional histogram shows the number of points for which a given $\phi^{\prime \prime}{ }_{\mathrm{w}}$ and $\phi^{\prime \prime \prime}{ }_{\mathrm{w}}$ value maximized the signal from a discrete 
region of emission; the bins correspond to the step size used, (50 $\mathrm{fs}^{2}$ and $1000 \mathrm{fs}^{3}$, respectively), chosen based on the experimental error. The results show a large range of values for quadratic and cubic dispersion introduced as the pulse propagates, encompassing both positive and negative values for each. The median value for quadratic dispersion was found to be $-100 \mathrm{fs}^{2}$, while the median value for cubic dispersion was found to be $0 \mathrm{fs}^{3}$. The magnitude and sign of the components of the dispersion do not exhibit any dependence on the position of the region in the sample; as indicated previously, adjacent regions may indicate the pulse has acquired very different values of dispersion.

\section{Conclusion}

In this paper, we have presented characteristics of SPP propagation to distances of tens of micrometers in silver nanoparticle covered quartz and mica substrates upon excitation with an ultrafast, near-IR laser. The nonlinear photoluminescence observed is consistent with the observed two-photon excitation of silver nanoparticle films and has characteristics very distinct from that of laser-scattered light. Upon measurement of the photoluminescence emitted by localized regions as a function of the delay between two replicas of the pulse, evidence for the acquisition of quadratic and cubic dispersion is observed, providing additional support to the suggestion that energy transport from the focal spot to the localized photoluminescent regions occurs through propagating SPPs. This dispersion is then quantified by determining the amount of prechirp phase required to optimize two-photon-induced luminescence. The system is found to have both positive and negative values, with no correlation observed between the location of the region of emission and acquired dispersion due to the randomness of the sample. The nature of the sample provides a number of paths for SPP transport, leading to the observed variations.

We propose the following hypothesis to explain our experimental observations. Silver nanoparticles with dimensions 20-100 nm, randomly arranged, with numerous interconnections and interparticle distances shorter than the optical wavelength, deposited on a dielectric surface provide a medium for long-range surface plasmon propagation. Femtosecond laser pulses with a central wavelength near $800 \mathrm{~nm}$ excite a surface plasmon resonance and launch a surface plasmon polariton on the very thin but interconnected layer of nanoparticles. The SPP, with a very long attenuation length, propagates from the excitation spot. The combination of constructive interference and localized surface plasmon resonance induces localized photoluminescence in the visible spectral range at distinct locations, which are observed as bright spots at distances of several tens of micrometers from the excitation point.

The observations presented here may inspire new strategies in which plasmonics can bridge nano- and microscopic systems.

Acknowledgment. The authors gratefully acknowledge discussions with Professor Mark Stockman. Partial support for this work came from funding for this research from the Chemical Sciences, Geosciences and Biosciences Division, Office of Basic Energy Sciences, Office of Science, U.S. Department of Energy.

Supporting Information Available: Further discussion of the MIIPS method for compensating ultrashort pulses, additional composite images to further demonstrate the spectral properties of the photoluminescence, and an elaboration on the validity of our approach to quantifying the spectral phase distortion acquired by the pulse. This material is available free of charge via the Internet at http://pubs.acs.org.

\section{References and Notes}

(1) Link, S.; El-Sayed, M. A. Annu. Rev. Phys. Chem. 2003, 54, 331. 131.

(2) Zayats, A.; Smolyaninov, I.; Maradudin, A. Phys. Rep. 2005, 408,

(3) Hartland, G. V. Annu. Rev. Phys. Chem. 2006, 57, 403.

(4) Sarkisov, O. Russ. Chem. Bull. 2008, 57, 736.

(5) Huang, W.; El-Sayed, M. Eur. Phys. J. Special Top. 2008, 153, 223. LP.

(6) Boyd, G. T.; Yu, Z. H.; Shen, Y. R. Phys. Rev. B 1986, 33, 7923

(7) Bouhelier, A.; Beversluis, M. R.; Novotny, L. Appl. Phys. Lett. 2003, 83, 5041 .

(8) Imura, K.; Nagahara, T.; Okamoto, H. J. Am. Chem. Soc. 2004, $126,12730$.

(9) Itoh, T.; Ozaki, Y.; Yoshikawa, H.; Ihama, T.; Masuhara, H. Appl. Phys. Lett. 2006, 88, 084102.

(10) Chen, C.; De Castro, A.; Shen, Y. Phys. Rev. Lett. 1981, 46, 145.

(11) Moskovits, M. Rev. Mod. Phys. 1985, 57, 783.

(12) Nie, S. M.; Emery, S. R. Science 1997, 275, 1102.

(13) Kneipp, K.; Wang, Y.; Kneipp, H.; Perelman, L. T.; Itzkan, I.; Dasari, R.; Feld, M. S. Phys. Rev. Lett. 1997, 78, 1667.

(14) Xu, H.; Bjerneld, E.; Käll, M.; Börjesson, L. Phys. Rev. Lett. 1999, 83,4357

(15) Eliel, E.; Van Der Ham, E.; Vrehen, Q. Appl. Phys. B: Lasers Opt. 1999, 68, 349 .

(16) Michaels, A.; Jiang, J.; Brus, L. J. Phys. Chem. B 2000, 104, 11965. (17) Johnson, R. C.; Li, J. T.; Hupp, J. T.; Schatz, G. C. Chem. Phys. Lett. 2002, 356, 534

(18) Gryczynski, I.; Malicka, J.; Shen, Y. B.; Gryczynski, Z.; Lakowicz,

J. R. J. Phys. Chem. B 2002, 106, 2191.

(19) Barnes, W. J. Opt. A: Pure Appl. Opt. 2006, 8, S87.

(20) Bozhevolnyi, S.; Pudonin, F. Phys. Rev. Lett. 1997, 78, 2823.

(21) Dickson, R.; Lyon, L. J. Phys. Chem. B 2000, 104, 6095.

(22) Charbonneau, R.; Berini, P.; Berolo, E.; Lisicka-Shrzek, E. Opt. Lett. 2000, 25, 844.

(23) Maier, S.; Brongersma, M.; Kik, P.; Meltzer, S.; Requicha, A.; Atwater, H. Adv. Mater. 2001, 13, 2.

(24) Maier, S.; Kik, P.; Atwater, H. Appl. Phys. Lett. 2002, 81, 1714.

(25) Ditlbacher, H.; Krenn, J. R.; Schider, G.; Leitner, A.; Aussenegg,

F. R. Appl. Phys. Lett. 2002, 81, 1762.

(26) Maier, S. A.; Kik, P. G.; Atwater, H. A. Phys. Rev. B 2003, 67.

(27) Haynes, C. L.; McFarland, A. D.; Zhao, L. L.; Van Duyne, R. P.;

Schatz, G. C.; Gunnarsson, L.; Prikulis, J.; Kasemo, B.; Kall, M. J. Phys.

Chem. B 2003, 107, 7337.

(28) Krenn, J.; Weeber, J. Philos. Trans. R. Soc. London, Ser. A 2004, 362,739 .

(29) Stockman, M. I. Phys. Rev. Lett. 2004, 93, 137404.

(30) Dionne, J. A.; Sweatlock, L. A.; Atwater, H. A.; Polman, A. Phys. Rev. B 2005, 72, 075405.

(31) Ozbay, E. Science 2006, 311, 189

(32) Citrin, D. S. Nano Lett. 2004, 4, 1561

(33) Citrin, D. S. Nano Lett. 2005, 5, 985.

(34) Citrin, D. S. Opt. Lett. 2006, 31, 98.

(35) Zou, S. L.; Schatz, G. C. Phys. Rev. B 2006, 74, 125111.

(36) Knight, M. W.; Grady, N. K.; Bardhan, R.; Hao, F.; Nordlander,

P.; Halas, N. J. Nano Lett. 2007, 7, 2346.

(37) Wei, W.; Li, S.; Qin, L.; Xue, C.; Millstone, J.; Xu, X.; Schatz, G.; Mirkin, C. Nano Lett. 2008, 8, 3446.

(38) Sarid, D. Phys. Rev. Lett. 1981, 47, 1927.

(39) Craig, A.; Olson, G.; Sarid, D. Opt. Lett. 1983, 8, 380

(40) Quail, J.; Rako, J.; Simon, H. Opt. Lett. 1983, 8, 377.

(41) Shalaev, V. M.; Sarychev, A. K. Phys. Rev. B 1998, 57, 13265.

(42) Yang, F.; Sambles, J.; Bradberry, G. Phys. Rev. B 1991, 44, 5855

(43) Lamprecht, B.; Krenn, J.; Schider, G.; Ditlbacher, H.; Salerno, M.;

Felidj, N.; Leitner, A.; Aussenegg, F.; Weeber, J. Appl. Phys. Lett. 2001, 79,51 .

(44) Ditlbacher, H.; Krenn, J. R.; Felidj, N.; Lamprecht, B.; Schider, G.; Salerno, M.; Leitner, A.; Aussenegg, F. R. Appl. Phys. Lett. 2002, 80, 404.

(45) Zou, S.; Schatz, G. J. Chem. Phys. 2004, 121, 12606.

(46) Maier, S. A.; Atwater, H. A. J. Appl. Phys. 2005, 98, 011101

(47) Murray, W. A.; Astilean, S.; Barnes, W. L. Phys. Rev. B 2004, 69, 165407.

(48) Oates, T.; Mücklich, A. Nanotechnology 2005, 16, 2606.

(49) Ding, S.; Wang, X.; Chen, D.; Wang, Q. Opt. Express 2006, 14, 1541.

(50) Gunn, J. M.; Ewald, M.; Dantus, M. Nano Lett. 2006, 6, 2804.

(51) Gong, H. M.; Xiao, S.; Su, X. R.; Han, J. B.; Wang, Q. Q. Opt.

Express 2007, 15, 13924. 
(52) Roberti, T.; Smith, B.; Zhang, J. J. Chem. Phys. 1995, 102, 3860. (53) Klar, T.; Perner, M.; Grosse, S.; von Plessen, G.; Spirkl, W.; Feldmann, J. Phys. Rev. Lett. 1998, 80, 4249.

(54) Lehmann, J.; Merschdorf, M.; Pfeiffer, W.; Thon, A.; Voll, S.; Gerber, G. Phys. Rev. Lett. 2000, 85, 2921.

(55) Kubo, A.; Onda, K.; Petek, H.; Sun, Z. J.; Jung, Y. S.; Kim, H. K. Nano Lett. 2005, 5, 1123.

(56) Bauer, M.; Wiemann, C.; Lange, J.; Bayer, D.; Rohmer, M.; Aeschlimann, M. Appl. Phys. A: Mater. Sci. Process. 2007, 88, 473.

(57) Raether, H. Surface Plasmons on Smooth and Rough Surfaces and on Gratings; Springer: Berlin, Germany, 1988; Vol. 111.

(58) Xu, B.; Gunn, J.; Cruz, J.; Lozovoy, V.; Dantus, M. J. Opt. Soc. Am. B 2006, 23, 750 .

(59) Wenseleers, W.; Stellacci, F.; Meyer-Friedrichsen, T.; Mangel, T.; Bauer, C. A.; Pond, S. J. K.; Marder, S. R.; Perry, J. W. J. Phys. Chem. B 2002, 106, 6853 .

(60) Merschdorf, M.; Pfeiffer, W.; Thon, A.; Voll, S.; Gerber, G. Appl. Phys. A: Mater. Sci. Process. 2000, 71, 547.

(61) Drachev, V. P.; Khaliullin, E. N.; Kim, W.; Alzoubi, F.; Rautian,

S. G.; Safonov, V. P.; Armstrong, R. L.; Shalaev, V. M. Phys. Rev. B 2004, 69, 036318.

(62) Bouhelier, A.; Bachelot, R.; Lerondel, G.; Kostcheev, S.; Royer,

P.; Wiederrecht, G. P. Phys. Rev. Lett. 2005, 95, 267405.

(63) Collier, C.; Saykally, R.; Shiang, J.; Henrichs, S.; Heath, J. Science 1997, 277, 1978

(64) Wu, Z.; Arakawa, E.; Inagaki, T.; Thundat, T.; Schowalter, L. Phys. Rev. B 1994, 49, 7782 .

(65) Takabayashi, M.; Haraguchi, M.; Fukui, M. J. Mod. Opt. 1997, $44,119$.

(66) Salomon, L.; Bassou, G.; Aourag, H.; Dufour, J.; de Fornel, F.; Carcenac, F.; Zayats, A. Phys. Rev. B 2002, 65, 125409.

(67) Berini, P.; Charbonneau, R.; Lahoud, N.; Mattiussi, G. J. Appl. Phys. 2005, 98, 043109.

(68) Gresillon, S.; Aigouy, L.; Boccara, A. C.; Rivoal, J. C.; Quelin,

X.; Desmarest, C.; Gadenne, P.; Shubin, V. A.; Sarychev, A. K.; Shalaev,

V. M. Phys. Rev. Lett. 1999, 82, 4520.
(69) Jiang, J.; Bosnick, K.; Maillard, M.; Brus, L. J. Phys. Chem. B 2003, 107, 9964.

(70) Burke, J.; Stegeman, G.; Tamir, T. Phys. Rev. B 1986, 33, 5186.

(71) Barnes, W. L.; Dereux, A.; Ebbesen, T. W. Nature 2003, 424, 824.

(72) Zolotavin, P.; Permenova, E.; Sarkisov, O.; Nadtochenko, V.; Azouani, R.; Portes, P.; Chhor, K.; Kanaev, A. Chem. Phys. Lett. 2008, 457, 342 .

(73) Kempa, T.; Farrer, R. A.; Giersig, M.; Fourkas, J. T. Plasmonics 2006, 1,45 .

(74) von Vacano, B.; Buckup, T.; Motzkus, M. J. Opt. Soc. Am. B 2007, 24,1091 .

(75) Galler, A.; Feurer, T. Appl. Phys. B 2008, 90, 427.

(76) Aeschlimann, M.; Bauer, M.; Bayer, D.; Brixner, T.; de Abajo, F. J.; Pfeiffer, W.; Rohmer, M.; Spindler, C.; Steeb, F. Nature 2007, 446, 301 .

(77) Fecher, G. H.; Schmidt, O.; Hwu, Y.; Schonhense, G. J. Electron Spectrosc. Relat. Phenom. 2002, 126, 77.

(78) Brongersma, M. L.; Hartman, J. W.; Atwater, H. A. Phys. Rev. B 2000, 62, R16356.

(79) Rokitski, R.; Tetz, K. A.; Fainman, Y. Phys. Rev. Lett. 2005, 95, 177401.

(80) Bai, M.; Guerrero, C.; Ioanid, S.; Paz, E.; Sanz, M.; García, N. Phys. Rev. B 2004, 69, 115416.

(81) Kubo, A.; Jung, Y. S.; Kim, H. K.; Petek, H. J. Phys. B: At., Mol. Opt. Phys. 2007, 40, S259.

(82) Stockman, M. I.; Faleev, S. V.; Bergman, D. J. Phys. Rev. Lett. 2002, 88, 067402 .

(83) Stockman, M. I.; Bergman, D. J.; Kobayashi, T. Phys. Rev. B 2004, 69,054202 .

(84) Stockman, M. I.; Hewageegana, P. Nano Lett. 2005, 5, 2325.

(85) Sukharev, M.; Seideman, T. Nano Lett. 2006, 6, 715.

(86) Li, X. T.; Stockman, M. I. Phys. Rev. B 2008, 77, 195109.

(87) Stockman, M. I. Phys. Rev. E 1997, 56, 6494.

(88) Durach, M.; Rusina, A.; Stockman, M. I. Nano Lett. 2007, 7, 3145. JP905319Q 